301 MANAGEMENT OF PATIENTS WITH ADVANCED STAGE EPITHELIAL OVARIAN CANCER: RESULTS OF A NATIONAL FRENCH SURVEY ON CURRENT PRACTICES IN 2021

${ }^{1} \mathrm{~L}$ Drouin*, ${ }^{2,3} \mathrm{~B}$ Guani, ${ }^{2} \mathrm{~V}$ Balaya, ${ }^{4} \mathrm{H}$ Azais, ${ }^{5} \mathrm{~S}$ Betrian, ${ }^{6} \mathrm{PA}$ Bolze, ${ }^{7} \mathrm{Y}$ Dabi, ${ }^{8} \mathrm{Y}$ Kerbage, ${ }^{9} \mathrm{C}$ Sanson, ${ }^{9} \mathrm{~F}$ Zaccarini, ${ }^{2,3} \mathrm{P}$ Mathevet, ${ }^{10} \mathrm{~F}$ Lécuru, ${ }^{11} \mathrm{~F}$ Guyon, ${ }^{12} \mathrm{C}$ Akladios, ${ }^{13} \mathrm{~S}$ Bendifallah, ${ }^{14} \mathrm{E}$ Deluche. ${ }^{1} \mathrm{CHU}$ de Limoges, Department of Gynecology, Limoges, France; ${ }^{2}$ CHUV, Gynecology , Lausanne, Switzerland; ${ }^{3}$ UNIL, Faculty of Biology and Medicine, Lausanne, Switzerland; ${ }^{4}$ Georges Pompidou European Hospital, Gynecologic and Breast Oncologic Surgery Department, Paris, France; ${ }^{5}$ IUCT Oncopole, Department of Médical Oncology, Toulouse, France; ${ }^{6} \mathrm{CHU}$ Lyon Sud, Department of Gynecology, Lyon, France; ${ }^{7}$ Tenon Hospital, AP-HP, Département of Gynecology, Paris, France; ${ }^{8}$ Jean de Flandre Hospital, Département of Gynecology, Lille, France; ${ }^{9}$ Gustave Roussy Institute, Surgical Oncology Department, Villejuif, France; ${ }^{10}$ Curie Institute, Breast. Gynecology and reconstructive surgery unit, Paris, France; ${ }^{11}$ Bergonié Institute, Surgical oncology Department, Bordeaux, France; ${ }^{12}$ University Hospital of Strasbourg, Department of Obstetrics and Gynecology, Strasbourg, France; ${ }^{13}$ Tenon Hospital AP-HP, Department of Gynecology, Paris, France; ${ }^{14} \mathrm{CHU}$ de Limoges, Department of Medical Oncology, Limoges, France

\subsection{6/ijgc-2021-ESG0.381}

Introduction/Background* The management of ovarian cancer has rapidly become more complex in recent years due to surgical and medical advances. The aim of this study was to assess French current practice in management of patients with advanced epithelial ovarian cancer.

Methodology An anonymous survey was sent to the SFOG, GINECO-ARCAGY and FRANCOGYN members. The survey consisted of 58 items divided in five sections dealing with diagnostic staging, pathological and genetic data, surgical practice patterns, adjuvant/neoadjuvant treatment, and follow-up strategies.

Result(s)* Overall, 107 answers were available. Most of participants were obstetrician-gynecologists (37.4\%), surgical oncologists (34.6\%), and medical oncologists (17.8\%). Most of participants came from University Hospitals (40.2\%) and Comprehensive Cancer Center (37.4\%).

For $76.8 \%$ of respondents, less than $50 \%$ of patients are eligible for primary debulking surgery. In case of neoadjuvant chemotherapy, surgical resecability is assessed after 3 cycles for $92.7 \%$ of respondents. LION study's criteria were always applied during primary surgery for $69,5 \%$ of respondents. Hyperthermic Intraperitoneal Chemotherapy (HIPEC) is used as a first-line treatment for $41.4 \%$ of respondents $(26.8 \%$

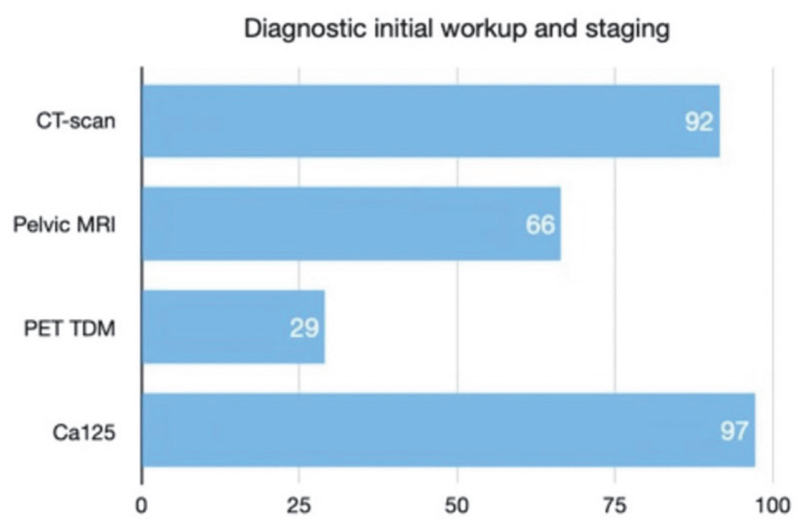

$\%$ of respondents

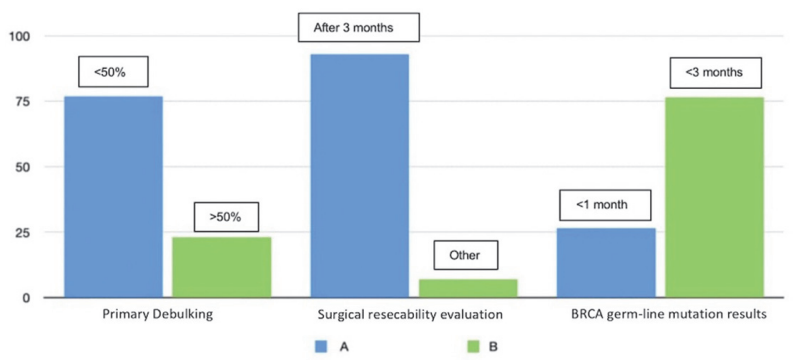

Abstract 301 Figure 2

within clinical trial and $14.6 \%$ outside) and during interval surgery for $37.8 \%$ of respondents.

According to the completeness of cytoreduction surgery and the BRCA mutation status, association of Paclitaxel-Carboplatin (every 3 weeks) is the most used combination as adjuvant chemotherapy for 69.7 to $84.8 \%$ of respondents and bevacizumab $15 \mathrm{mg} / \mathrm{kg}$ is used in case of no residual disease for 52.9 to 61.7 of respondents.

In case of BRCA1-2 mutations, Olaparib is given by $75.8 \%$ to $84.8 \%$ of respondents. The delay to obtain BRCA germline mutation results is less than 1 month for $26.5 \%$ of respondents and less than 3 months for $76.5 \%$ of respondents. An Homologous Recombination Repair Deficiency is searched for $73.5 \%$ of respondents and mainly by Myriad test (91.3\%).

Conclusion* In our study, the rate of HIPEC was high because 2 clinical trials are currently ongoing in France on HIPEC as 1 st line treatment.

Our results provide an interesting picture of current management in France in relation to International Guidelines. They provide a basis for further research and raise the question of updating guidelines for ovarian cancer management.

\section{RELATIONSHIP AMONG SURGICAL COMPLEXITY, EARLY AND LATE MORBIDITIES, AND SURGICAL TIMING IN ADVANCED OVARIAN CANCER}

${ }^{1} \mathrm{D}$ Ferraioli*, ${ }^{2} \mathrm{~L}$ Fuso, ${ }^{3} \mathrm{~F}$ Beurrier, ${ }^{1} \mathrm{P}$ Meeus, ${ }^{4} \mathrm{H}$ Lorraine, ${ }^{1} \mathrm{R}$ Ray-Coquard, ${ }^{1} \mathrm{~N}$ Chopin. 'Léon Bérard Center, Lyon, France; ${ }^{2}$ Mauriziano Umberto Hospital, Torino, Italy; ${ }^{3}$ Caluire-etCuire, Surgical Oncology, Caluire-et-Cuire, France; ${ }^{4}$ Institute Cancer De Lorraine - Alexis Vautrin, Vandouvre-lès-Nancy, France

\subsection{6/ijgc-2021-ESG0.382}

Introduction/Background* Epithelial ovarian cancer (EOC) requires an aggressive surgical approach. The type and the number of procedures performed during primary (PDS) or interval (IDS) debulking surgery in order to achieve no residual disease are associated with considerable morbidities.

The objective is to describe a relationship among surgical complexity, early and late morbidities, and surgical timing in advanced EOC.

Methodology A retrospective study was performed at Leon Berard Cancer Center between 2006 and 2018. Surgical complexity was classified into three groups (standard, radical and ultra-radical surgery) based on the type and the number of procedures performed during PDS or IDS for advanced EOC. During the 30- and 90-day period after the surgery, the postoperative complications were registered according to the Clavien-Dindo classification. 
Result(s)* 311 patients with advanced EOC were included. 84 patients underwent PDS (27\%) and 227 IDS (73\%), respectively.

No residual disease was achieved in 258 out of 311 patients $(83 \%)$. Standard surgery was performed in 80 patients $(25.7 \%)$, radical surgery in 45 patients $(14.5 \%)$ and ultra-radical surgery in 186 patients $(59.8 \%)$. No residual disease was reached in 72 out of 80 standard surgery (90\%), 37 out of 45 radical surgery $(82.2 \%)$ and 149 out of 186 (80.1\%) ultraradical surgery.

Early and late severe (G3-4) complications were present in $32 / 311$ patients $(10.3 \%)$ and $23 / 311$ patients (7.4\%), respectively. Increased postoperative morbidity was directly related to the following perioperative factors: HIPEC [OR 4.4 (1.0$20)$ ], addition of each single surgical procedures to the standard surgery [OR $1.3(1.1-1.5)]$, number of surgical procedures added to modified pelvic posterior exenteration [OR 1.4 (1.12.0)], surgical complexity [SR vs $\mathrm{S}$ or R; OR 2.4 (1.1-5.7)].

In multivariate analysis, OS is not correlated with surgical complexity [OR 1.0(0.5-2.1)] and with the burden of early $[$ OR $0.7(0.3-1.5)]$ or late $[\mathrm{OR} 0.7(0.3-1.7)]$ severe complications.

Conclusion* Despite surgical timing, ultra-radical-surgery is needed to reach no residual disease in $>80 \%$ of our patients. The risk of early and late severe morbidities is increased for complex surgeries but they don't affect OS. Risk stratification should be used to plan perioperative care and best treatment.

\section{ERAS LEADS TO FEWER HOSPITAL COSTS IN ADVANCED OVARIAN CANCER SURGERY: A SECONDARY OUTCOME OF THE PROFAST TRIAL}

\begin{abstract}
${ }^{1} V$ Bebia Conesa*, 'S Rodríguez Conde, ${ }^{1} \mathrm{~PB}$ Asuncion, ${ }^{2} \mathrm{Y}$ Cossío Gil, ${ }^{1} \mathrm{~A}$ Gil-Moreno, ${ }^{2} J \mathrm{~L}$ Sánchez Iglesias. 'Hospital Universitari Vall d'Hebron, Gynecologic Oncology Unit, Barcelona, Spain; ${ }^{2}$ Hospital Universitari Vall d'Hebron, Information Systems, Barcelona, Spain
\end{abstract}

10.1136/ijgc-2021-ESG0.383

Introduction/Background* Enhanced recovery after surgery (ERAS) programs in advanced ovarian cancer (AOC) surgery have been developed in the last few years. The PROFAST randomized controlled trial showed that ERAS was associated with a decrease on length of stay and readmission. Here, we present the results of a secondary outcome, cost analysis, of the PROFAST trial.

Methodology The PROFAST trial was a prospective, interventional randomized clinical trial that enrolled women undergoing surgery for either suspected or diagnosed advanced ovarian cancer, at a reference hospital in gynecologic oncology in Barcelona (Spain). Patients were treated following either an ERAS protocol or conventional management (CM) protocol. The primary outcome was to evaluate reduction in length of stay (LOS). One of the secondary outcomes was to perform cost analysis betweem both groups.

Cost estimations were performed by two strategies: gross counting (cost of inpatient care, intensive care unit and surgery care), and microcosting (costs associated to image testing and laboratory). In both cases, costs were based on 2014 cost data. A sensitivity analysis of cost savings in different scenarios was performed. This trial was registered at ClinicalTrials.gov (NCT02172638).

Result(s)* From June 2014 to March 2018, 110 women were recruited, of which eleven were excluded. The ERAS group comprised 50 patients, and the CM group, 49 patients. LOS. Although mean overall cost per patient was higher in ERAS vs CM (14347 $€$ vs $12597 €)$, ERAS cost distribution was skewed by two patients that accounted for $48 \%$ of total intensive care unit costs. Comparing median costs, ERAS costs were significantly lower than CM costs $(7642 € /$ patient vs $8594 € /$ patient, $\mathrm{P}=.0275)$. Decrease in hospital readmission rates led to higher readmission costs in the $\mathrm{CM}$ group (Total costs $6126 € \mathrm{~V} 125725 €, \mathrm{P}=.028)$. Sensitivity analysis

\begin{tabular}{|cccc} 
Abstract 315 Table 2 & \\
\cline { 2 - 4 } & ERAS group & Conventional group & Difference \\
\hline Worst scenario & 9 median days & 12 median days & 3 median days \\
& $3,507 €$ & $4,654.01 €$ & $-\mathbf{1 1} 146.63 €$ \\
Best scenario & 5 median days & 7 median days & 2 median days \\
& $1,949 €$ & $2,714.84 €$ & $-\mathbf{7 6 6 . 3 0 €}$ \\
\hline
\end{tabular}

\begin{tabular}{|c|c|c|c|c|c|c|c|c|c|}
\hline \multirow[b]{2}{*}{ Resources } & \multicolumn{4}{|c|}{ ERAS (n: 50 patients) } & \multicolumn{5}{|c|}{ Conventional group (n: 49 patients) } \\
\hline & Total & Mean & Median & IQR & Total & Mean & Median & IQR & p-value* \\
\hline \multicolumn{10}{|l|}{ Cost in wards } \\
\hline First hospitalization in ward & $180.888 €$ & $3.618 €$ & $2.848 €$ & $2.085 €$ & $204.255 €$ & $4.169 €$ & $3.280 €$ & $2.193 €$ & 0,0588 \\
\hline Readmission day in ward & $5.894 €$ & $118 €$ & $0 €$ & $0 €$ & $59.303 €$ & $1.210 €$ & $0 €$ & $0 €$ & 0,0287 \\
\hline Total cost & $186.783 €$ & $3.736 €$ & $2.849 €$ & $2.098 €$ & $263.558 €$ & $5.379 €$ & $3.430 €$ & $3.021 €$ & 0,0143 \\
\hline \multicolumn{10}{|l|}{ Surgical costs } \\
\hline Primary surgical program & $195.669 €$ & $3.913 €$ & $3.638 €$ & $1.012 €$ & $184.997 €$ & $3.775 €$ & $3.697 €$ & $1.022 €$ & 0,3253 \\
\hline Reinterventions & $0 €$ & $0 €$ & $0 €$ & $0 €$ & $10.750 €$ & $219 €$ & $0 €$ & $0 €$ & 0,0771 \\
\hline Total cost & $195.669 €$ & $3.913 €$ & $3.638 €$ & $1.012 €$ & $195.747 €$ & $3.995 €$ & $3.697 €$ & $1.070 €$ & 0,2312 \\
\hline \multicolumn{10}{|l|}{ Image test and lab } \\
\hline Image test and lab at first hospitalization & $31.789 €$ & $662 €$ & $231 €$ & $499 €$ & $19.650 €$ & $401 €$ & $238 €$ & $468 €$ & 0,5421 \\
\hline Image test and lab at readmissions & $322 €$ & $6 €$ & $0 €$ & $0 €$ & $4.607 €$ & $94 €$ & $0 €$ & $0 €$ & 0,0455 \\
\hline Total cost & $32.111 €$ & $642 €$ & $205 €$ & $474 €$ & $24.257 €$ & $495 €$ & $265 €$ & $490 €$ & 0,1673 \\
\hline \multicolumn{10}{|l|}{ ICU cost } \\
\hline Total ICU cost at first hospitalization & $302.761 €$ & $6.582 €$ & $925 €$ & $350 €$ & $82.633 €$ & $1.836 €$ & $964 €$ & $233 €$ & 0,7209 \\
\hline Total ICU cost at readmissions & $0 €$ & $0 €$ & $0 €$ & $0 €$ & $51.065 €$ & $1.042 €$ & $0 €$ & $0 €$ & 0,151 \\
\hline Total cost & $302.761 €$ & $6.055 €$ & $900 €$ & $337 €$ & $133.698 €$ & $2.729 €$ & $964 €$ & $296 €$ & 0,6093 \\
\hline \multicolumn{10}{|l|}{ Total cost of inpatient resources } \\
\hline Total cost first hospitalizaton & $711.108 €$ & $14.222 €$ & $7.642 €$ & $3.062 €$ & $491.535 €$ & $10.031 €$ & $8.236 €$ & $4.015 €$ & 0,089 \\
\hline Total cost readmissions & $6.216 €$ & $124 €$ & $0 €$ & $0 €$ & $125.725 €$ & $2.569 €$ & $0 €$ & $0 €$ & 0,0287 \\
\hline Total cost & $717.324 €$ & $14.347 €$ & $7.642 €$ & $3.062 €$ & $617.260 €$ & $12.597 €$ & $8.594 €$ & $4.693 €$ & 0,0275 \\
\hline
\end{tabular}

